\title{
Participación Social y Política de Personas Mayores Chilenas: Un Estudio Exploratorio desde la Perspectiva Narrativa-Generativa*
}

\section{Social and Political Participation of Chilean Older People: An Exploratory Study from the Narrative-Generative Perspective}

DOI: $10.46919 / \operatorname{archv} 2 n 8-003$

Recebimento dos originais: 01/11/2021

Aceitação para publicação: 27/12/2021

\section{Eduardo Sandoval-Obando}

Grupo de Investigación en Cultura, Educación, y Transformación Social (ICET), Escuela de Psicología,

Facultad de Ciencias Sociales y Humanidades, Universidad Autónoma de Chile (Chile).

Psicólogo, Magíster en Educación; Doctor en Ciencias Humanas. Investigador responsable del

FONDECYT de Iniciación $\mathrm{N}^{\circ} 11190028$. Académico adscrito a la Escuela de Psicología, Facultad de

Ciencias Sociales y Humanidades, Universidad Autónoma de Chile (Chile).

E-mail: eduardo.sandoval.o@gmail.com

\section{Valentina Altamirano}

Grupo de Investigación en Cultura, Educación, y Transformación Social (ICET), Escuela de Psicología, Facultad de Ciencias Sociales y Humanidades, Universidad Autónoma de Chile (Chile).

\section{Bruno Isla}

Grupo de Investigación en Cultura, Educación, y Transformación Social (ICET), Escuela de Psicología, Facultad de Ciencias Sociales y Humanidades, Universidad Autónoma de Chile (Chile).

\section{Valeska Loyola}

Grupo de Investigación en Cultura, Educación, y Transformación Social (ICET), Escuela de Psicología, Facultad de Ciencias Sociales y Humanidades, Universidad Autónoma de Chile (Chile).

\section{Camila Painecura}

Grupo de Investigación en Cultura, Educación, y Transformación Social (ICET), Escuela de Psicología, Facultad de Ciencias Sociales y Humanidades, Universidad Autónoma de Chile (Chile).

\section{RESUMEN}

En Chile, se observa un cambio sociodemográfico relevante como consecuencia del proceso de envejecimiento acelerado de la población, lo que ha incidido en la disminución de las tasas de natalidad y al aumento en la esperanza de vida. Además, la pandemia provocada por el virus SARS-CoV-2 ha generado diversas problemáticas para las personas durante los periodos prolongados de confinamiento. Esta investigación tuvo como proposito comprender e interpretar el potencial generativo desplegado por personas mayores no institucionalizadas que residen en Temuco (Chile) y que participan activamente en organizaciones socio-comunitarias, develando las implicaciones de estas actividades para su calidad de vida en contexto de pandemia. Metodológicamente, la investigación se construyó desde el paradigma cualitativo, siguiendo un diseño descriptivo, no experimental transversal. Se utilizó una muestra no probabilítica, de tipo intencional conformada por 8 personas mayores autovalentes, que participan regularmente en

\footnotetext{
* Trabajo financiado por la Agencia Nacional de Investigación y Desarrollo (ANID) / FONDECYT de Iniciación en Investigación No 11190028 "La Profesionalidad Docente Rural: Implicaciones Socioeducativas desde la Perspectiva Narrativa Generativa”.
} 
organizaciones sociales, comunitarias y/o políticas. Para la recolección de los datos, se utilizaron entrevistas en profundidad desde la perspectiva narrativa (Sandoval-Obando, 2019). Para el análisis de contenido se siguió la lógica de la teoría fundamentada (Strauss y Corbin, 2002). Los resultados evidencian que los participantes han desplegado acciones potencialmente generativas que le han permitido enfrentar de manera funcional y propositiva la pandemia, potenciando un sentido de vida que fortalece el deseo de mantenerse activos en la esfera social y comunitaria. Finalmente, dicho potencial generativo devela una alta capacidad para reinventarse ante los desafíos y obstáculos suscitados durante la pandemia.

Palabras claves: Generatividad, Envejecimiento, Pandemia, Calidad de Vida, Sentido de Vida.

\begin{abstract}
In Chile, a relevant sociodemographic change is observed as a consequence of the accelerated aging process of the population, which has had an impact on the decrease in birth rates and an increase in life expectancy. In addition, the pandemic caused by the SARS-CoV-2 virus has created various problems for people during prolonged periods of confinement. The purpose of this research was to understand and interpret the generative potential displayed by non-institutionalized older people who reside in Temuco (Chile) and who actively participate in socio-community organizations, revealing the implications of these activities for their quality of life in the context of a pandemic. Methodologically, the research was built from the qualitative paradigm, following a descriptive, not cross-sectional experimental design. A non-probabilistic sample was used, of an intentional type, made up of 8 self-supporting older people, who regularly participate in social, community and / or political organizations. For data collection, in-depth interviews were used from the narrative perspective (Sandoval-Obando, 2019). For the content analysis, the logic of grounded theory was followed (Strauss and Corbin, 2002). The results show that the participants have deployed potentially generative actions that have allowed them to face the pandemic in a functional and purposeful way, enhancing a sense of life that strengthens the desire to remain active in the social and community sphere. Finally, this generative potential reveals a high capacity to reinvent itself in the face of the challenges and obstacles raised during the pandemic.
\end{abstract}

Keywords: Generativity, Aging, Pandemic, Quality of Life, Meaning of Life.

\title{
1 INTRODUCCIÓN
}

El proceso de envejecimiento enfrenta ritmos acelerados en Chile y el mundo, lo que obliga a generar procesos de investigación sistemáticos que respondan a las necesidades, intereses y desafíos que enfrentan las personas mayores (Villalobos, 2019). Al respecto, Arias e Iglesias (2015) afirman que la generatividad funciona como un mecanismo adaptativo, aportando beneficios en torno a los indicadores de bienestar y salud, lo que permite reflexionar acerca de la importancia de desarrollar investigaciones que visibilicen los intereses, experiencias y prácticas cotidinadas de las personas mayores, develando las diversas implicaciones que emergen a partir de un desarrollo potencialmente generativo.

En esta investigación se abordó la forma en que las personas mayores vivencian y perciben su calidad de vida, relacionándolo con lo propuesto por McAdams y de St. Aubin (1992), quienes plantean que la generatividad estaría compuesta por ciertas características psicosociales que podrían incidir positivamente en la forma en la que afrontan esta etapa del ciclo vital. Además de repercutir en un mayor 
nivel de bienestar y en una mejora directa en su calidad de vida. Al respecto, la calidad de vida es descrita como aquel "bienestar general que comprende descriptores objetivos y evaluaciones subjetivas de los aspectos físicos, materiales, sociales y bienestar emocional junto con el grado de desarrollo personal y actividad intencional, todo ello ponderado por un conjunto de valores personales" (Karimi y Brazier, 2016, p.3).

Una de las consecuencias de la pandemia fueron "el aislamiento social, la movilidad restringida y el pobre contacto con los demás, ha incrementado la vulnerabilidad en las personas mayores a presentar múltiples alteraciones psicológicas" (Callis et al., 2021, p.2), por consiguiente, el estudio se orientó a comprender e interpretar el potencial generativo manifestado por las personas mayores de Temuco-Chile, en contexto de pandemia y las implicancias que esto tiene para su calidad de vida. Del mismo modo, nos interesó profundizar en cómo esta característica puede repercutir en sus percepciones con respecto al envejecimiento, el sentido de vida y la calidad de vida en el complejo contexto socio-sanitario actual.

La generatividad como constructo no sólo conlleva "procrear y criar hijos, incluye otras esferas en las que se puede expresar la preocupación generativa: actividades profesionales, participación en organizaciones sociales, políticas o religiosas, activismo vecinal y comunitario, tareas de voluntariado y relaciones de amistad" (Villareal et al., 2020, p.47). Además, el comportamiento generativo es complejo y heterogeneo, pudiendo manifestarse de diversas formas. Es decir, opera como un "marco de trabajo amplio que comprende diversas actividades y contextos no sólo muy relevantes desde un punto de vista científico, sino también con un indudable interés social y político" (Villar, 2012, p.47).

La generatividad surge como un recurso prometedor al considerar los cambios en la sociedad actual, y por ello se define como "la preocupación y el compromiso de un adulto por promover el crecimiento y bienestar de las generaciones futuras a través de la crianza, la enseñanza, la tutoría, la participación institucional, y una amplia variedad de comportamientos sociales" (McAdams, 2013, p.11). La generatividad es considerada, para los autores Sandoval-Obando et al. (2019), como una herramienta de gran importancia e indispensable para la etapa evolutiva, dando trascendencia a las personas mayores como sujetos autovalentes y con capacidades de adaptación a los distintos contextos y cambios que surgen, siendo sujetos que contribuyen a la sociedad a través de su experiencia y saberes acumulados a lo largo de la vida (Riquelme Brevis y Sandoval-Obando, 2021).

La generatividad es un campo innovador y complejo que debe ser analizado en detalle, especialmente en la población mayor de Chile, ya que forma parte imprescindible del proceso de envejecimiento. Al respecto, Ercoli y Sandoval-Obando (2021) refieren que el comportamiento y desarrollo generativo en la vejez "demuestra que algunas de las acciones, tareas, actividades y esfuerzos desplegados durante el envejecimiento, estarían relacionadas con un desarrollo generativo", siguiendo esta línea resulta interesante comprender cómo se desarrolla y visualiza el envejecimiento en Chile, especialmente en la 
región de la Araucanía puesto que "la generatividad se puede expresar a partir de actividades muy variadas: la implicación activa de mayores en la vida cívica, política, social, asociacionismo y voluntariado" (Ercoli y Sandoval-Obando, 2021).

Por consiguiente, esta investigación tuvo como proposito comprender e interpretar el potencial generativo desplegado por personas mayores no institucionalizadas que residen en Temuco (Chile) y que participan activamente en organizaciones socio-comunitarias, develando las implicaciones de estas actividades para su calidad de vida en contexto de pandemia. En este sentido, nos interesó explorar el comportamiento potencialmente generativo desplegado por las personas mayores, operando como un constructo relevante para la comprensión de la experiencia vital durante el afrontamiento de episodios vitales estresantes. Esta revisión nos permite tener una visión más amplia acerca del desarrollo de esta etapa del ciclo vital, integrando experiencias, saberes y prácticas que tributen a mejorar los niveles de bienestar y de calidad de vida en las personas mayores chilenas.

El estudio de la generatividad en personas mayores ofrece una comprensión más amplia acerca del envejecimiento y del importante rol que cumplen las personas mayores en la sociedad, reafirmando la necesidad de cuestionar los estereotipos socialmente vinculados a la vejez, dando el salto hacia una perspectiva que los reconozca como sujetos activos, funcionales e interesados a seguir enfrentando su vida de manera óptima y saludable. (González-Celis y Mendoza, 2016).

Cabe señalar que el comportamiento generativo está sumamente relacionado con el desarrollo y el bienestar en la adultez (Baranski et al., 2020). Así, es posible destacar que la generatividad se expresaría en "la preocupación y el compromiso de un adulto por promover el crecimiento y bienestar de las generaciones futuras a través de la crianza, la enseñanza, la tutoría, la participación institucional, y una variedad de otros comportamientos sociales" (McAdams, 2013, p.11).

Es importante destacar que esta investigación se enmarcó en la ejecución del proyecto FONDECYT de Iniciación No 11190028 "La Profesionalidad Docente Rural: Implicaciones Socioeducativas desde la Perspectiva Narrativa Generativa" ejecutado por el Dr. Eduardo Sandoval Obando, gracias al financiamiento de la Agencia Nacional de Investigación y Desarrollo (ANID) / Ministerio de Ciencia, Tecnología, Conocimiento e Innovación de Chile.

\section{MÉTODO}

\subsection{DISEÑO DEL ESTUDIO}

La presente investigación se ejecutó bajo el paradigma cualitativo siguiendo un diseño de tipo descriptivo, no experimental y transversal, teniendo como propósito comprender e interpretar el potencial generativo desplegado por personas mayores no institucionalizadas que residen en la comuna de Temuco 
(Chile) y que participan activamente en organizaciones socio-comunitarias, develando las implicaciones de estas actividades para su calidad de vida en contexto de pandemia.

\subsection{PARTICIPANTES}

Los participantes fueron escogidos a través de un muestreo no probabilístico de tipo intencional. Para la obtención de los datos requeridos, se contactó a 8 personas mayores residentes en la ciudad de Temuco que participen en agrupaciones de índole social, comunitario y/o político, cumpliendo con los criterios de inclusión y exclusión definidos para esta investigación (ver tabla 1).

Tabla 1 Criterios de inclusión y exclusión de los Participantes

\begin{tabular}{cc}
\hline Criterios de inclusión & Criterios de exclusión \\
\hline Participación voluntaria en el estudio. & $\begin{array}{l}\text { Personas mayores con dependencia moderada y } \\
\text { severa que le impidan la movilidad y participación en } \\
\text { actividades sociales. }\end{array}$ \\
\hline $\begin{array}{c}\text { Personas mayores con una edad igual o superior } \\
\text { a } 60 \text { años. }\end{array}$ & $\begin{array}{l}\text { Personas mayores con trastornos mentales } \\
\text { incapacitantes que le impidan la comprensión y/o }\end{array}$ \\
\hline $\begin{array}{c}\text { Temuco, región de La Araucanía. } \\
\text { interal. }\end{array}$ & $\begin{array}{l}\text { Personas mayores que tengan un nivel de } \\
\text { discapacidad que los invalide para comunicarse de } \\
\text { manera oral, así como aquellos que no hablen el idioma } \\
\text { español. }\end{array}$ \\
\hline
\end{tabular}

Personas mayores que utilizan las redes de apoyo social presente en la comuna de Temuco, región de La Araucanía.

Personas mayores que, antes de la pandemia, participaran regularmente (asisten una o más veces al mes) en una organización socio comunitaria presente en la comuna de Temuco. Personas mayores con alta vulnerabilidad

Personas mayores con alta vulnerabilidad
social, en situación de calle o abandono.

Personas mayores que cumpliendo con los criterios de inclusión, no tienen disponibilidad para participar en la investigación.
Personas mayores que sean polivalentes y que no presenten antecedentes de comorbilidad grave.
Personas mayores que cumpliendo con los criterios de inclusión, no residan en la comuna donde se realizará la investigación.

No hay distinción por género, así como tampoco por nivel socioeconómico, escolarización o nacionalidad.

Nota: Fuente de elaboración propia.

El levantamiento de datos se realizó durante los meses de abril y mayo del año 2021. Así, la muestra quedó conformada por 8 personas mayores residentes en la comuna de Temuco (Chile), autovalentes, de las cuales un $75 \%$ eran mujeres $(n=6)$ y un $25 \%$ hombres ( $n=2$ hombres), con una edad promedio de 75,5 años. Con respecto a su estado civil, un 50\% de los participantes declararon estar casados/as ( $\mathrm{n}=4$ ), un 37,5 eran viudos/as $(n=3)$ y sólo un $12,5 \%$ convivía con su pareja. Por otra parte, la mayoría de los sujetos se 
encuentran jubilados (75\%), a diferencia de algunos (25\%) que declararon mantenerse activos laboralmente en actividades relacionadas con la construcción y/o coordinación política local.

\subsection{TÉCNICA DE RECOLECCIÓN DE DATOS}

Para el proceso de levantamiento de datos, se utilizó la entrevista en profundidad desde la perspectiva narrativa generativa, la cual implica un proceso de análisis de datos en el que se contemplarán los relatos e historias de vida de las personas mayores a través de sus vivencias y significados (SandovalObando, 2019). Como plantea Kvale (2011) estos relatos se centran en lo que las personas narran desde su propio contexto, historias y estructuras de sus propios relatos. Estas narraciones surgen en el momento que se lleva a cabo la entrevista o bien el propio entrevistador puede provocarlas.

Al utilizar este tipo de entrevista, emerge como un tema esencial las narrativas que las personas mayores construyen para dotar de significado sus experiencias vitales (McAdams, 2001). La generatividad se convierte en un constructo relevante para comprender las historias de vida que han orientado a las personas mayores. Este enfoque en particular podría caracterizarse como "aquel relato retrospectivo de la experiencia vital de un sujeto, en donde el objeto de estudio es el potencial generativo" (Sandoval-Obando, 2020, p.285). A partir de esta investigación se busca profundizar en los relatos, experiencias, saberes y prácticas cotidianas desplegadas por personas mayores no institucionalizadas que residen en la comuna de Temuco (Chile) y que participan activamente en organizaciones socio-comunitarias, develando las implicaciones potencialmente generativas de estas actividades para su calidad de vida en contexto de pandemia.

\subsection{ESTRATEGIA DE ANÁLISIS E INTERPRETACIÓN DE LOS DATOS}

Para el análisis de los datos, se recurrió al análisis de contenido, siguiendo la lógica de la teoría fundamentada (Strauss y Corbin, 2002), la cual consiste en "una teoría derivada de datos recopilados de manera sistemática y analizados por medio de un proceso de investigación. En este enfoque, la recolección de datos, el análisis y la teoría que surgirá de ellos guardan estrecha relación entre sí" (p.21), permitiendo ordenar y delimitar la información obtenida a través de las entrevistas realizadas a las personas mayores, lo que proporcionará algún grado de estandarización y rigor al proceso (Strauss y Corbin, 2002).

Respecto al resguardo de los criterios de rigor científico, la ejecución del presente estudio se ejecutó fundado sobre los siguientes criterios: relevancia, credibilidad, consistencia y confirmabilidad. Además, desde el punto de vista ético, se asegura el anonimato, confidencialidad y privacidad en el tratamiento de la información, por tal razón en lo sucesivo nos referiremos a los participantes con nombres ficticios: Gloria, Leonor, Luis, Antonio, Orlando, Adela, Berta y Alicia. 


\section{RESULTADOS}

Tras el análisis e interpretación de los datos, es posible inferir que los obstáculos provocados por la pandemia influyeron en que los/as participantes desplegaran acciones potencialmente generativas para el afrontamiento de los eventos estresantes suscitados durante los periodos prolongados de confinamiento. Del mismo modo, pareciera ser que un sentido de vida sólido y flexible, favoreció el interés generativo de las personas mayores por mantenerse activos en las organizaciones socio-comunitarias en las que participan, buscando contribuir al desarrollo y bienestar de otros/as.

Las vivencias y experiencias de las personas mayores permitieron develar la emergencia de seis macro-categorías (ver tabla 2): características propias de las personas mayores, conducta generativa en personas mayores, calidad de vida orientada por acciones potencialmente generativa, redes de apoyo de las personas mayores en contexto de pandemia, sentido de vida potencialmente generativo en personas mayores y desafíos y proyecciones generativas en personas mayores.

Tabla 2 Categorías y Códigos generados a partir de las entrevistas transcritas:

\begin{tabular}{|c|c|}
\hline Categorías & Códigos \\
\hline $\begin{array}{l}\text { Características } \\
\text { Propias de las Personas } \\
\text { Mayores }\end{array}$ & $\begin{array}{l}\text { Edad, Actividad productiva, Estado civil, Diversidad familiar, Entretenimiento } \\
\text { en la vejez, Autopercepción Rol en la vejez. }\end{array}$ \\
\hline $\begin{array}{l}\text { Conducta } \\
\text { Generativa en Personas } \\
\text { Mayores }\end{array}$ & $\begin{array}{l}\text { Actividad generativa, Participación en organizaciones, Legado intelectual y } \\
\text { valórico, Sabiduría en la vejez, Motivación comunitario, Liderazgo generativo, Aprendizaje } \\
\text { autodidacta, Autogestión, Flexibilidad en la vejez, Actividad comunitaria, Logro } \\
\text { comunitario, Resiliencia. }\end{array}$ \\
\hline $\begin{array}{l}\text { Calidad de Vida } \\
\text { Orientada por Acciones } \\
\text { Potencialmente } \\
\text { Generativas }\end{array}$ & $\begin{array}{l}\text { Dependencia económica, Estimulación gerontomotriz, Vulnerabilidad social, } \\
\text { Reinventarse en pandemia, Deterioro físico, Deterioro Cognitivo, Autocuidado, } \\
\text { Sentimiento de soledad, Beneficios de la participación social, Geronto acción, Actividad } \\
\text { física antes de la pandemia, Autopercepción de la etapa vital, Vida en pandemia, Capacidad } \\
\text { autovalente, Envejecimiento primario, Geronto salud, Disminución en la autonomía, } \\
\text { Enfermedades preexistentes, Ejercicio en confinamiento. }\end{array}$ \\
\hline
\end{tabular}

Redes de Apoyo de las Personas Mayores en Contexto de Pandemia
Reciprocidad entre pares, Valores transgeneracionales, Apoyo gubernamental durante la contingencia sanitaria, Apoyo gubernamental antes de la contingencia sanitaria, Soporte familiar en la vejez, Redes sociales como apoyo, Orgullo familiar, Añoranza de vida social, Amor a la comunidad, Resignificación del duelo, Comunidad comprometida.
Sentido de Vida Potencialmente Generativo en Personas Mayores
Altruismo, creencia espiritual, Sentido de vida, Cuidado transgeneracional, Valores transgeneracionales, Visión social, Satisfacción personal.

Desafíos y $\quad$ Proyección a futuro, Sentimiento de desesperanza, Recambio generacional.
$\begin{aligned} & \text { Proyecciones Generativas } \\ & \text { en Personas Mayores }\end{aligned}$

Nota. La tabla representa los códigos y categorías obtenidas a partir de las narrativas de las personas mayores no institucionalizadas en contexto de la pandemia Temuco - Chile. 
En lo que respecta a la conducta generativa en personas mayores, destaca la motivación comunitaria, donde se identifica el caso de Leonor, Gloria, Orlando y Alejandro que tienen en común el que participan en el mismo centro de personas mayores llamado "Amistad 3000" en donde el compartir, ayudar y generar momentos con un otro son claves en la motivación que ellos identifican, en donde expresan una sensación de gratificación mediante anécdotas o recuerdos de su grupo, destacándose como principal motivante la ayuda social a otros, esto se puede ejemplificar en el caso de Gloria, que refiere "Yo me siento feliz cuando ellos proponen vamos a tal parte, vamos al otro, cómo lo hacemos, con qué plata, entonces ahí hay que trabajar para lograr que te acepten el proyecto y hacer cositas, vender para que te acepten el proyecto", como también en la narrativa de Leonor que expresa: "Uno tiene una preocupación, que tenía que asistir y cooperar, si eso era, más que cooperar, ayudar”.

La autogestión, entendida como aquel conjunto de prácticas y acciones cotidianas que despliegan las personas mayores de manera autónoma para generar algún tipo de beneficio personal o grupal, resulta clave para entender la conducta generativa, puesto que en la totalidad de los casos las personas mayores se han visto obligadas a aceptar las medidas y limitaciones que implica el confinamiento, siendo la autogestión un importante aliado para poder adaptarse al contexto actual. Leonor en relación a esto ha identificado labores que puede desempeñar como hacer conservas, tejer o criar plantas sin la necesidad de salir fuera de su hogar, si bien antes de la pandemia, la participante desempeñaba estas labores, en la actualidad la puede hacer con mayor agrado y tiempo, una de las limitantes que detecta Leonor a sus 81 años es que no puede salir a supermercados, puesto que por su edad no la dejan entrar, sin embargo la comunicación fluida y activa con sus hijos le ha permitido un desarrollo funcional de sus actividades cotidianas.

En el contexto actual de pandemia, las personas mayores como Luis, Alejandro, Gloria, Leonor, Berta y Orlando han debido sobrellevar grandes limitantes producto de su edad, sin embargo la resiliencia entendida como la capacidad individual para adaptarse y sobreponerse a situaciones adversas, ha sido determinante para comprender sus experiencias especialmente en este contexto de confinamiento, es así como también la resiliencia resulta clave para comprender cómo las personas mayores han sobrellevado las dificultades de tipo económico, salud física y emocional a lo largo de su ciclo vital, en el caso de Berta; "Para mí fue muy duro perdí mi casa, porque en ese tiempo lo mismo que ahora enfrentarse a un cáncer muy caro así que fue realmente muy duro, tenía que salir adelante, ponerles el pecho a las balas y salir adelante, primero soy madre y las mujeres tenemos más fuerza que los hombres, será porque parimos a un hijo".

Respecto a la calidad de vida guiada por la acción generativa, los relatos de las personas mayores permiten construir esta categoría representada como aquel marco de elementos objetivos y subjetivos que configuran la percepción acerca de la calidad de vida individual y colectiva del grupo etario. Suele estar permeada por elementos histórico-culturales, sociales y económicos, mientras exista un acceso a estos 
elementos, la acción generativa, que puede aparecer como un factor protector de la calidad de vida de la persona mayor, extrapolable a diversas situaciones y contextos cotidianos.

Las personas mayores residentes en Temuco (Chile) que han dado vida a esta investigación, otorgan relevancia a la participación que tienen en diversos grupos sociales, ya sea de índole político o sociocomunitario, debido a los beneficios personales que esto trae consigo, que si bien no son percibidos como una retribución material en las actividades sociales que realizaban, les aporta diversos beneficios en su salud mental, tales como sentirse mejor, optimistas, alegres, felices y satisfechos personalmente. Al respecto, Antonio menciona "Entonces sí, la actividad trajo mucha satisfacción personal el creer que era algo valioso y era más valioso porque ad honorem. Sí eso es lo valioso, era un voluntariado”, Orlando "En el grupo súper bien, me siento feliz, la vida se me hace más amena” y Adela; “ Yo me siento bien, me gusta, me gusta participar y estar en el grupo es bonito".

Considerando el contexto actual de pandemia, que ha provocado el cese de las actividades sociales potencialmente generativas que influyen en la calidad de vida de las personas mayores, se debe tener en consideración que una de las consecuencias de la contingencia sanitaria tiene repercusión en la salud mental de las personas. Tal como relata Leonor "Porque yo me siento muy, muy sola sobre todo en la tarde que ya, se oscurece tan temprano y no pueden venir los hijos, las nietas... ”. Por su parte, Gloria señala “Hemos estado tanto en cuarentena aquí en Temuco, entonces eso me deprime y me ha quitado como mi manera de ser..." expresan que debido a la pandemia sus relaciones interpersonales se han visto afectadas y así también el cese de las actividades grupales, situación que les hace sentir solos y afectados por el confinamiento, interpretándolo como una percepción de calidad de vida más baja a la que se presenta cuando pueden realizar actividades de índole social, recreativas o de su cotidianeidad.

Reinventarse en pandemia es una habilidad que le ha permitido a los participantes adaptarse a los desafíos y condiciones de vida generadas por el confinamiento, avanzando en la incorporación de nuevas maneras de relacionarse e interactuar con otras personas a través del uso de programas tecnológicos como Zoom. En este sentido, Berta menciona "Yo empecé primero con él con el teléfono, pero todavía me da el cuero para para aprender, la verdad creo que la modalidad online va a durar mucho tiempo”, dentro del mismo contexto de acciones nuevas para reinventarse en pandemia con el fin de mantenerse activos y saludables, Adela refiere que ante la falta de actividad social o de otras índoles, se reinventa a través de las actividades de cocina, "Ahora poquito porque tengo que hacer para uno, pero trato a veces de copiar algunas recetas de la tele y trato de hacer algunas comidas distintas”. Se desprende de lo anterior, la existencia de una alta flexibilidad y autogestión de las personas mayores frente a la pandemia, permitiéndoles integrar nuevas formas de comunicación y de aprendizajes con el propósito de mantenerse activos en el ámbito social, cognitivo y emocional. 
En referencia a las redes de apoyo de las personas mayores en contexto de pandemia, estas tienen a la base un conjunto de factores de índole social, emocional y afectivo que confluyen sinérgicamente para la comunicación, participación e integración socio-comunitaria. A partir de dichas instancias, surgen sentimientos, pensamientos y acciones basadas en la interacción y colaboración, que pueden actuar como factor protector o de riesgo para las personas mayores, en relación a esto cabe destacar que las redes de apoyo cumplen un factor fundamental en la vida de las personas mayores, esto se logra comprobar en entrevistas como la de Berta, en donde la buena comunicación con su familia, colegas de trabajo y clientes la motivan a seguir trabajando hasta el día de hoy, lo que hace con gusto y no solo por necesidad.

El compañerismo entre las personas mayores operan como un soporte social positivo para la participación socio-comunitaria, como en el caso de Gloria, en donde ella es la presidenta de un centro de adulto mayor y gracias a la buena comunicación con los miembros ha logrado ganar proyectos y compartir el beneficio de estos con su grupo y parte de la comunidad, siendo una figura significante y querida por su comunidad. Además, se rememora la añoranza de una vida social activa. Al respecto, Gloria señala "me gustaría, por ejemplo, poder compartir con otras personas, yo tengo una amiga, que nos vemos un poquito, pero eso, juntarme con otras personas y conversar", se identifica claramente el interés social que tiene Gloria por compartir con otras personas además del amor a su comunidad.

En otro ámbito, los participantes describen con pesar el bajo apoyo gubernamental disponible para las diversas necesidades que enfrentan las personas mayores en Chile. Al respecto, Orlando menciona que "De la parte de la municipalidad, nos mandaron una caja, de lo demás cero y del gobierno el aporte IFE (ingreso familiar de emergencia) que le llaman de emergencia, nada más, pensé que iba a recibir el de quinientos por la clase media, pero nada" y Luis: "Una vez no más, sí, del resto de las otras cosas no nada, ni bonos ni nada", indicando así que tienen grandes limitaciones en torno a la obtención de bonos como el IFE o el de clase media y las pensiones son muy bajas, por lo que deben buscar la forma de obtener ingresos de otras fuentes. Tal es el caso de Orlando quien ha arrendado algunas de sus propiedades o en el caso de Berta quien se mantiene activamente trabajando, ya que si se quedaran sólo con ayudas estatales, no lograrían cubrir sus necesidades básicas.

Otro de los campos temáticos emergentes giran en torno a la generatividad como guía en el sentido de vida de las personas mayores. Dicha macro-categoría se construye a partir del significado personal que orienta y moviliza las acciones y decisiones de una persona a lo largo de su vida. Este significado individual y particular tiene a la base creencias, metas, valores y perspectivas, cabe destacar que la generatividad, en relación con el sentido de vida, suele orientar las acciones y metas de la persona para generar aportes en la esfera social y comunitaria, así como el apoyo hacia terceros, con tal de acercarse al bienestar social. Todas estas acciones y motivaciones generan en el individuo una sensación de satisfacción y orgullo, al ver que su conducta tributa a mejoras colectivas. 
Relacionado directamente con el reconocimiento y la satisfacción personal, se da paso al altruismo, el cual guía las acciones sociales de Gloria, donde refiere "Yo, en principio lo compartía con los chicos haitianos, los migrantes, pero también hay personas aqui en el barrio que necesitan” y Leonor que comenta "Teníamos que ir a ver a alguna persona enferma y así cosas". Quienes comentan que antes y durante la contingencia sanitaria, han interactuado de manera desinteresada con personas en situación de vulnerabilidad: migrantes haitianos, vecinos o participantes del grupo de adultos mayores en situaciones de enfermedad o con necesidades económicas, agudizadas por la situación actual. Suelen participar en estas actividades de manera individual, aunque, cuando la situación lo requiere, recurren a sus redes de apoyo para brindar un apoyo integral a las personas que lo requieren.

Cabe destacar que en la mayoría de los participantes se observa una orientación marcada hacia la promoción de valores transgeneracionales potencialmente generativos, el cual se ve reflejado en el cuidado hacia las generaciones más jóvenes, sean estos familiares, amigos y/o conocidos. Asimismo, es relevante el legado intelectual y valórico que buscan transmitirle a las generaciones más jóvenes como es el caso de Berta quien señala "el hecho de poder contar toda esta historia a mis hijos, a mis nietos las historias que yo he ido pasando" y Gloria quien entregar su conocimiento "Así es que ahí volví a retomar como la cosa de enseñar, de educar, porque aparte de enseñarles a hablar a ellos también se les entregan valores como a todos, en la educación eso es primordial. Eso", destacando la importancia de transmitir un conocimiento hacia nuevas y futuras generaciones en base a sus propias experiencias de vida.

En cuanto a las creencias espirituales, estas constituyen una guía importante en la conducta de los entrevistados y, por ende, fortalece el sentido de vida de las personas mayores. La mayoría de los entrevistados, con excepción de Leonor y Antonio, "Qué más le vamos a pedir a Dios, la salud que nos de no más, que tenga buena salud", hacen referencia a la benevolencia y gracia de Dios hacia sus vidas, remitiendo, en ocasiones, que su calidad de vida, salud física y mental es producto de la gracia de Dios. De la misma manera, proyectan sus deseos de futuro, individuales y sociales, en línea con su credo religioso; "Yo soy católica cristiana, apostólica, romana y entonces yo rezar, pedir al señor, a la virgen, que cambie, que haya un cambio que la gente piense un poquito, que no sé qué se mejore ojalá, que con la oración se hace mucho, hay que saber pedirselo también". Respecto a este último punto, manifiestan un deseo de mantener sus actuales condiciones de vida, en términos de autonomía, salud óptima, condiciones económicas estables y las interacciones sociales con sus pares.

En conexión con lo anteriormente descrito, es relevante la proyección a futuro de personas mayores en contexto de pandemia fundada sobre las perspectivas individuales de los participantes, muchas de las cuales se co-construyen con el paso del tiempo, las dinámicas relacionales que cultivan y sus ideas de futuro. De igual manera, se amplían a través del intercambio de ideas, principios y valores que observan en las nuevas generaciones. No obstante, igual manifiestan la existencia de sentimientos de soledad al imaginar 
o pensar situaciones sociales futuras, lo que se relaciona directamente con el contexto actual de pandemia, ya que en entrevistas como la de Gloria; "Sabes que yo siempre pienso, que sí salimos bien de esta pandemia, porque quizás cuando va a terminar, si no logro... yo no voy a alcanzar a ver todos los planes que hay, para el futuro del país, por ejemplo, eso de las asambleas constituyentes, el cambio de la constitución, yo pienso que no lo voy a ver, no voy a ver los cambios que van a hacer, ya sea para mejor o peor", no ven un cambio a corto plazo, percibiendo el período de confinamiento con diversas limitaciones y carencias a futuro, lo que impacta negativamente en su calidad de vida.

Con respecto a las proyecciones de futuro de las personas mayores, se destaca el interés generativo de mantenerse activos en el plano socio-comunitario, lo que se relaciona con sus creencias y deseos cotidianos de seguir vinculados a las organizaciones en las que participan como una manera de contribuir al bien común y al desarrollo de sus comunidades. De hecho, en algunos casos el contexto actual de pandemia ha modificado la proyección a futuro que tenían originalmente respecto al envejecimiento, puesto que en palabras de Gloria, ella espera que exista un recambio generacional: "Y vendrán otros que traen otras ideas. Porque la gente más joven, una persona de 60 años piensa muy diferente a lo que yo pienso. Entonces, a lo mejor, todos los clubes se van a ir renovando, rejuveneciendo quizás" reafirmando su deseo de enfrentar la vida de manera activa, saludable y en comunidad.

\section{DISCUSIÓN}

Desde las narrativas aportadas por los participantes de estudio, se pudo inferir que las pautas de comportamiento evidencian un conjunto de acciones y prácticas potencialmente generativas, vinculadas a los roles de voluntariado y participación activa en organizaciones socio-comunitarias y políticas, a pesar de los periodos de confinamiento y restricciones sanitarias implementadas durante la pandemia. Lind et al. (2021) sostienen que, al haber vivido grandes retos personales en momentos difíciles e históricos, serían capaces de tener una mayor perseverancia ante el COVID-19, debido a la historia de vida que han construido, llegando así a prosperar incluso frente a las adversidades que se presentan en la actualidad. Si bien se mencionó que la generatividad se ha visto potencialmente afectada en las personas mayores, estos también han sido capaces de sobreponerse a estas dificultades, modificando sus rutinas cotidianas y replanteándose las dinámicas relacionales para mantenerlas mediante el uso de dispositivos tecnológicos (videollamadas, SMS, redes sociales, etc.).

Para Sandoval-Obando et al. (2019) las personas mayores son "sujetos independientes, capaces de adaptarse a los diversos cambios que la vida presenta y continuar aportando con su experiencia en la construcción de una sociedad inclusiva, diversa y tolerante" (p.22), aludiendo y respaldando los resultados encontrados de esta investigación. La capacidad de adaptación presente en este grupo etario salió a relucir en este contexto histórico y sanitario, a pesar de las complejidades y limitaciones, lograron adaptarse 
siguiendo su vida a pesar de las medidas sanitarias. Es así como lograron mantenerse activas en el ámbito socio-comunitario, siendo este un importante espacio de reinvención y soporte social entre pares. Esta acción comunitaria va de la mano con la resiliencia, que ha sido una característica de este grupo y que ha funcionado como factor protector durante este período, permitiéndoles mantener sus estados de salud y estado de ánimo estable (MacLeod et al., 2016). Para algunos autores como Gorbea y Piña (2021), la resiliencia actúa como promotor de calidad de vida, protegiéndola ya que está es habilidad psicosocial y fundamental que permite e impulsa a la persona a poder sobrellevar y enfrentarse a las adversidades que se presentan y así, recuperarse de las consecuencias negativas que esto trae consigo y se ha descrito que mientras mayor sea el nivel de resiliencia, será menor el padecimiento o susceptibilidad de padecimiento de enfermedades psicológicas.

Aún así, la calidad de vida de las personas mayores se ha visto afectada, puesto que la pandemia impactó negativamente en sus prácticas cotidianas, particularmente en el plano físico y psicológico, siendo coherente con lo reportado por Ribeiro, Morais y Fernandes (2021) quienes en una revisión, identificaron la presencia de cambios de humor, aumento de los niveles de ansiedad / depresión y diversos síntomas psicosomáticos. A su vez, Sandoval-Obando y Sandoval-Díaz (2020) han señalado que las cuarentenas preventivas y las diversas restricciones a las libertades de las personas, se convierten en un factor de riesgo para la salud mental de las personas, particularmente para los grupos de riesgo (entre ellos, las personas mayores). El confinamiento, como expresan Mera, Tabares, Montoya, et. al. (2020) "redunda en incremento de la inactividad física y de comportamientos sedentarios, favoreciendo el desacondicionamiento físico. Las personas desacondicionadas físicamente tienen alteraciones metabólicas y sistémicas por la falta de movimiento".

Los participantes de esta investigación, han dejado de participar en instancias tales como controles de salud en la atención primaria (tratamientos médicos de enfermedades crónicas no transmisibles, kinesiólogos, terapeutas ocupacionales, examenes, etc.), repercutiendo en su salud física, al reducir considerablemente la movilidad. Es decir, los niveles de sedentarismo han aumentado como consecuencia de los periodos de inactividad durante las cuarentenas sanitarias, impactando en la fuerza muscular y capacidades cardiorrespiratorias (Márquez, 2020).

En otro ámbito, sería plausible inferir que el desarrollo generativo se refuerza a través del interés, preocupación y comunicación con otro/a, en donde el apoyo familiar, vecinal y/o comunitario han sido claves para fortalecer las dinámicas relacionales a pesar del distanciamiento físico. Concepción-Bretón, Corrales-Camacho, Córdoba et.al. (2020) mencionan que el uso de dispostivos tecnológicos para mantenerse unidos en pandemia ha favorecido positivamente estos vínculos, observándose que un $73 \%$ reconoció que ha hecho mayor uso de las TIC a partir del confinamiento, fundamentalmente para 
comunicarse con familiares, amigos y grupos de pares, a través de chat (whatsapp), video-llamadas y el uso de las redes sociales.

Otra de las dimensiones estudiadas dentro del comportamiento generativo son los valores transgeneracionales y aprendizajes que comparten con sus grupos de pares. A pesar de la contingencia, siguen desplegando intereses potencialmente generativos relacionados con la vida en comunidad, mediante el uso de plataformas como Zoom o WhatsApp, además de las llamadas telefónicas con familiares y amigos, independientemente de que el uso de estos dispositivos tecnológicos han sido una tarea desafiante y novedosa (Galeano, 2020). Todas estas acciones les permiten mantenerse en contacto con sus seres significativos y seguir activos dentro de la comunidad en que se encuentran insertos, permitiéndoles fortalecer sus redes de apoyo, mediante una vida social y/o laboral activa. No obstante, es necesario enfatizar que este grupo etario suelen ser los menos capacitados digitalmente, siendo más vulnerables frente al uso de estas tecnologías, levantando un conjunto de beneficios, desafíos y posibilidades para la salud digital (CEPAL, 2020), en la medida que se adapten dichas tecnologías y plataformas a las necesidades e intereses de las personas mayores como una respuesta gubernamental con enfoque en los derechos humanos (Forttes, 2020).

La espiritualidad es un factor influyente en la configuración del sentido de vida de las personas mayores entrevistadas, ya que refuerza el espíritu altruista, la convicción y motivación de ser un aporte hacia otros/as, conectándose con un desarrollo potencialmente generativo durante el proceso de envejecimiento. De la misma forma, este ímpetu hacia el cuidado de terceros les permite alcanzar su propio desarrollo humano (Loaiza, 2005), encontrando razones por las cuales vivir y construir su propia vida, tanto en lo individual como en lo colectivo. Ejemplos de estas acciones se observa en tareas de voluntariado, participación activa en organizaciones sociales (tales como grupos de personas mayores y juntas de vecinos, entre otras). La espiritualidad no necesariamente debe estar ligada a una religión, sino que es mucho más amplia y que lo sagrado no sólo abarcaría a dios y entes superiores (Ramírez y Serra, 2020). Siguiendo esta línea se debe tener en consideración el sentido de vida, especialmente en personas mayores puesto que "mantener o formular proyectos que les ayuden a conectarse con sus deseos, motivaciones y capacidades generando un estado de Bienestar y satisfacción con su vida se hace necesario para que las personas mayores puedan continuar esta etapa de manera natural" (Neira, 2021, p.19).

Según Archilla et al. (2017) las personas mayores tienen metas claras a futuro que se pueden plasmar en diferentes actividades, tales como cultivar un hobbies, retomar una carrera, desarrollar una actividad placentera, etc. Es así, que resultan ser un aporte significativo hacia su comunidad, su familia y a sus cercanos, al dejar un legado mediante la transmisión de sus experiencias y saberes a las generaciones más jóvenes, contribuyendo a su propio autodesarrollo. Resaltamos la importancia de que las personas mayores logren vivenciar sus últimos años de vida, con buenas condiciones, en espacios que les permitan 
desarrollarse y afrontar los desafíos futuros en plenitud. El fortalecimiento de las redes familiares y círculos de cuidado robustecidos, serían una de las alternativas necesarias para la valoración y reconocimiento de las personas mayores en nuestra sociedad. Esto se relaciona directamente con los resultados obtenidos en este estudio, en donde las proyecciones a futuro de las personas mayores se encuentran estrechamente ligadas a sus figuras significativas, como familiares, grupo de pares y comunidades de origen. La generatividad implica ser un factor movilizador para las personas mayores, desde brindar apoyo, cuidado y soporte a un otro de manera simbólica; transmitir los conocimientos, saberes y experiencias a las futuras generaciones, la participación política y/o social, el compromiso cívico con la sociedad hasta fortalecer sus metas y anhelos mediante un desarrollo claro del sentido de vida, dimensiones que en su conjunto, favorecen un desarrollo potencialmente generativo que redunda en efectos beneficiosos para los gerontes, tales como tener un estilo de vida más activo, mejor salud y una satisfacción vital generalizada (Larraín, Zegers y Orellana, 2019).

Los indicadores muestran que las personas mayores son las más afectadas por la pandemia no solo por el virus, sino que también por las desigualdades sociales estructurales imperantes en Chile. Para Osorio et al. (2021) "la crisis por Covid-19 en Chile profundizó las desigualdades ya existentes en la vejez y develó la real situación de precariedad de las condiciones de vida de las personas mayores bajo el modelo económico neoliberal” (p.231). Este es uno de los hallazgos importantes de la investigación, puesto que la situación de vulnerabilidad que se visualizó en este grupo etario, han afectado sus rutinas y prácticas cotidianas, disminuyendo las reuniones presenciales, además del aporte (económico, sanitario y/o cultural) superficial del Estado en la satisfacción de sus necesidades básicas. En este sentido, se suele observar en la vejez el abandono de parte del Estado hacia las personas mayores, como el incumplimiento con lo necesario para las pensiones y bonos dirigidos a este grupo etario. Ortega (2018) plantea que el abandono "se produce cuando cualquier persona o institución no asume la responsabilidad que le corresponde en el cuidado del adulto mayor, o que habiendo asumido el cuidado o custodia de un adulto mayor lo desampara de manera voluntaria" (p.226). Por consiguiente, sería plausible inferir que el Estado no ha cumplido con la responsabilidad brindarle condiciones dignas a las personas mayores. Sin embargo, a pesar de la falta de apoyo estatal, el potencial generativo observado en los participantes les ha permitido afrontar funcionalmente la pandemia, sumado al hecho de que las familias de los gerontes han contribuido a que tengan mejores condiciones de vida en el plano económico, sanitario y socio-emocional.

En otro ámbito, destacamos el estudio de la generatividad como una linea de investigación promisoria y desafiante en torno a los recursos y potencialidades que poseen las personas mayores. Al respecto, "la generatividad (en tanto, mecanismo adaptativo), se relaciona con un mayor grado de bienestar y ajuste social durante el envejecimiento, destacando el papel de los y las gerontes como proveedores de recursos y no como una carga, transformándola en una tarea psicosocial relevante dentro del ciclo vital" 
(Mendoza, 2017, como se cita en Sandoval-Obando, 2019). Asimismo, la perspectiva generativa sería relevante y pertinente en el contexto chileno, puesto que favorecería "el reconocimiento de las personas mayores como sujetos independientes, capaces de adaptarse a los diversos cambios que la vida le presenta y continuar aportando con su experiencia en la construcción de una sociedad inclusiva, diversa y tolerante", dando paso a una mirada más humana y optimista respecto al envejecimiento (Sandoval-Obando, 2018).

\section{CONCLUSIÓN}

La generatividad es un campo temático promisorio y relevante para comprender los desafíos, tareas y crisis evolutivas que enfrentan las personas adultas, especialmente en el proceso de transición a la adultez tardía (Sandoval-Obando, Serra y Zacarés, 2019; Sandoval-Obando y Zacarés, 2020). El impacto de la pandemia y restricciones sanitarias han tenido consecuencias en las pautas potencialmente generativas que desplegaron las personas mayores. No obstante, es posible inferir que los participantes han generado un afrontamiento potencialmente generativo frente las situaciones adversas suscitadas en el contexto sociosanitario actual. Esta capacidad de adaptación puede verse reflejada en conductas que cultivan la participación activa en organizaciones socio-comunitarias y el apoyo mutuo entre pares. Del mismo modo, frente al distanciamiento físico han incorporado positivamente los dispositivos tecnológicos para el fortalecimiento de los vínculos con sus familiares, amigos y comunidades de origen.

Finalmente, destacamos en los participantes el optimismo, flexibilidad y apertura al aprendizaje con que han afrontado la pandemia, puesto que a pesar de que reconocen la existencia de emociones negativas como ansiedad, miedo y tristeza frente a los obstáculos y limitaciones instaladas durante los periodos prolongados de confinamiento, éstas no lograron paralizar sus roles, acciones y tareas generativas. Al contrario, las personas mayores han tenido una respuesta adaptativa ante las implicancias de la pandemia, a través de la resiliencia, el sentido de vida y el deseo de sentirse parte de las comunidades a las que pertenecen. Los elementos descritos en este trabajo, dan cuenta de un conjunto de dimensiones, factores y pautas de comportamiento potencialmente generativas que inciden favorablemente en una adaptación positiva y óptima frente al proceso de envejecimiento, aportando saberes y experiencias relevantes para el estudio y comprensión de estos procesos desde la perspectiva de la psicología del ciclo vital. 


\section{REFERENCIAS}

Álvarez, C., Sanhueza, M., Valenzuela, T. y León, A. (2020). Factores asociados al envejecimiento, su asociación con SARS-CoV-2 e Impacto de la Pandemia en Personas Mayores. Revista Chilena Medicina Familiar, $14(1)$

5-14.

https://www.revistachilenademedicinafamiliar.cl/index.php/sochimef/article/view/361/338

Archilla, M., Rodriguez, J. y Archilla, M. (2017). Mejora de las capacidades cognitivas en personas mayores. En Molero, M., Perez, C., Gázquez, J,. Barragan, A., Martos, A., y Simón, M. (Eds.), Asunivep. Salud y cuidados durante el desarrollo (145-147). Asociación Universitaria de Educación y Psicología.

Baranski, E., Lodi-Smith, J., Ponterio, E., Newton, N., Poulin, M. y Whitbourne, S. (2020). The co-development of generativity and well-being into early late life. https://doi.org/10.31219/osf.io/5jbzp

Callis, S., Guarton, O., Cruz, V. y De Armas, A. (2021). Manifestaciones psicológicas en adultos mayores en aislamiento social durante la pandemia COVID-19. Segundo congreso virtual de ciencias básicas biomédicas, Granma, Cuba. https://aniversariocimeq2021.sld.cu/index.php/ac2021/Cimeq2021/paper/viewFi\%20le/73/50

Comisión Económica para América Latina y el Caribe. (2020). Universalizar el acceso a las tecnologías $\begin{array}{lllll}\text { digitales para enfrentar los efectos del } & \text { COVID-19. }\end{array}$ https://repositorio.cepal.org/bitstream/handle/11362/45938/S2000550_es.pdf?sequence=4\&isAllowed=y

Concepción-Breton, A. Corrales-Camacho, I. Córdoba, M., Acosta-Hernández, M., Larancuent-Cueto, O. y De la Cruz-Morel, Y. (2020). Sondeo de casos en personas mayores sobre actividades cotidianas y utilización de tecnologías de la información y la comunicación (TIC) en tiempos de pandemia. Revista Internacional Tecnológica-Educativa Docentes, 9(2), 132-150. https://doi.org/10.37843/rted.v9i2.156

Curcio, C. (2020). Ser persona mayor en tiempos de pandemia. En C. Robledo (Ed.), La Vejez (81-98). FUNDACOL.

Ercoli, K. y Sandoval-Obando, E. (2021). Implicaciones del Envejecimiento Potencialmente Generativo en Personas Mayores Chilenas: Una Exploración Inicial/ Implications of Potentially Generative Aging in Chilean Older People: An Initial Exploration. Brazilian Journal of development, 7(11), 9-18. https://doi.org/10.34117/bjdv7n11-226

Forttes, P. (2020). COVID-19, crisis y cambio social. En C. Robledo (Ed.), La Vejez (99-112). FUNDACOL.

Galeano, M., Zuluaga, M. y Saldarriaga, G. (2020). Envejecimiento y Covid-19: los debates que impone el confinamiento de los mayores a un mundo envejeciente. En Uribe et al. (Ed.) Polifonía para pensar una pandemia (145-166). Universidad de Antioquia, Fondo Editorial FCSH de la Facultad de Ciencias Sociales y Humanas.

Gorbea, S. y Piña, M. (2021). Investigación y metría de la información sobre COVID-19: Diversos enfoques de la pandemia. (1era Ed.) Instituto Nacional de Salud Pública.

Gutierrez, L. (2020). Salud mental en adultos mayores relacionada al aislamiento ocasionado por COVID-19. https://medicina.uniandes.edu.co/sites/default/files/articulos/doc/salud-mental-en-adultos-mayoresrelacionada-al-aislamiento-por-covid-19.pdf

Kvale, S. (2011). Las entrevistas en investigación cualitativa. Morata.

Larraín, M., Zegers, B. y Orellana, Y. (2019). Generatividad y satisfacción vital en una muestra de abuelos/as de Santiago, Chile. Terapia Psicológica, 37(3), 271-185. http://dx.doi.org/10.4067/S0718-48082019000300271 
Lind, M., Bluck, S. y McAdams, D. (2021). More Vulnerable? The Life Story Approach Highlights Older People's Potential for Strength During the Pandemic. The Journals of Gerontology, 76(2), 45-48. https://academic.oup.com/psychsocgerontology/article/76/2/e45/5875103?login=true

Loaiza, O. (2005). Construcción del sentido de vida en jóvenes universitarias [Tesis de Doctorado]. Universidad Iberoamericana.

MacLeod, S., Musich, S., Hawkins, K., Alsgaard, K. y Wicker, E. (2016). The impact of resilience among older adults. Geriatric Nursing, 266-272. https://reader.elsevier.com/reader/sd/pii/S0197457216000689?token=82B9AA31CD3305CB1B7F9FE93A7D E6603D4848223CB65DBCFE67ACB2D2C8820374D4D203932C63091E8C00E01E5DE149\&originRegion =us-east-1\&originCreation $=20210625221143$

Márquez, J. (2020). Inactividad física, ejercicio y pandemia COVID-19. Revista de Educación Física, 9(2), 43 56. https://revistas.udea.edu.co/index.php/viref/article/view/342196/20802578

McAdams, D. (2001). The psychology of life stories. Review of General Psychology, 5(2), 100-122.

McAdams, D. (2013). The Positive Psychology of Adult Generativity: Caring for the Next Generation and Constructing a Redemptive Life. En J. Sinnot (Ed.), Positive Psychology (191-205). Springer Science + Business Media.

Meléndez, J., Fortuna, F., Sales, A. y Mayordomo, T. (2015). The effects of instrumental reminiscence on resilience and coping in elderly. Archives of Gerontology and Geriatrics, 60(2), 294-298. https://www.sciencedirect.com/science/article/abs/pii/S0167494314002258?via\%3Dihub

Mera, A., Tabares-Gonzalez, E., Montoya-Gonzalez, S., Muñoz-Rodriguez, D., \& Monsalve Vélez, F. (2020). Recomendaciones prácticas para evitar el desacondicionamiento físico durante el confinamiento por pandemia asociada a COVID-19. Universidad Y Salud, 22(2), 166-177. https://doi.org/10.22267/rus.202202.188

Neira, N. (2021). Sentido de vida y calidad de vida en personas mayores residentes en el gran Concepción [Tesis de Magister]. Universidad de Concepción. http://repositorio.udec.cl/xmlui/handle/11594/6710

Observatorio del Envejecimiento. (2020). Medidas nacionales e internacionales de confinamiento y desconfinamiento en los 60+. https://observatorioenvejecimiento.uc.cl/wpcontent/uploads/2020/09/Reporte medidas desconfinamiento.pdf

Observatorio del Envejecimiento. (2020). Personas mayores en contexto de pandemia y aislamiento social. https://media.elmostrador.cl/2020/05/aislamiento social.pdf

Ortega, D. (2018). Envejecimiento y trato hacia las personas mayores en Chile: una ruta de la desigualdad persistente. Sophia Austral, (22), 223-246. https://dx.doi.org/10.4067/S0719-56052018000200223

Osorio-Parraguez, P., Jorquera, P. y Araya, M. (2021). Vejez y vida cotidiana en tiempos de pandemia: $\begin{array}{lllll}\text { estrategias, decisiones } & \text { y } & \text { cambios. } & \text { Artigos, } & \text { 27(59), }\end{array}$ https://www.scielo.br/j/ha/a/kgRD8yW9zsV6yxq4rwqvjNJ/?lang=es

Ramírez, M. y Serra, E. (2020). Espiritualidad en la vejez: perdón, gratitud y sentido de la vida. En E. SandovalObando., E. Serra y O. García. (Eds.), Nuevas miradas en psicología del ciclo vital (299-328). RiL editores. https://repositorio.uautonoma.cl/bitstream/handle/20.500.12728/6924/sandoval_digital.pdf?sequence=1\&isAll owed =y 
Ribeiro, A., Morais, J. y Fernandes, M. (2021). Enfrentamento à pandemia pela COVID-19 e os impactos na saúde mental da população: uma revisão narrativa: Coping with the pandemic by COVID-19 and the impacts on the population's mental health: a narrative review. Archives of Health, 2(4), 1268-1271. Retrieved from https://latinamericanpublicacoes.com.br/ojs/index.php/ah/article/view/619

Riquelme Brevis, H. y Sandoval Obando, E. (2021). Prácticas de movilidad y desempeño laboral del profesorado rural en la Región de La Araucanía, Chile. Revista Transporte Y Territorio, (24), 32-55. Recuperado de http://revistascientificas.filo.uba.ar/index.php/rtt/article/view/10226

Sandoval-Obando, E. (2018). Envejecimiento en Chile: Una Discusión Necesaria. El Mostrador. Recuperado de http://www.elmostrador.cl/agenda-pais/2018/07/20/envejecimiento-en-chile-una-discusion-necesaria/

Sandoval-Obando, E. (2019). La Profesionalidad Docente Rural: Implicaciones Socioeducativas desde la Perspectiva Narrativa Generativa. FONDECYT de Iniciación No 11190028. Temuco: Documento sin publicar.

Sandoval-Obando, E., Serra, E. y Zacarés, J. (2019). Envejecer Generativamente: Una Propuesta desde el Modelo del Buen Vivir. Neurama. Revista Electrónica de Psicogerontología, 6(2): 16-26. Recuperado de http://46.29.49.21/ creanete/neu/articulos/articulo2.pdf.

Sandoval-Obando, E. (2020). Caracterizando la Identidad Narrativa en Educadores Rurales Chilenos: Una Propuesta de Investigación. En E. Sandoval-Obando, E. Serra Desfilis y Ó. García. Nuevas Miradas en Psicología del Ciclo Vital (pp. 273-298). Santiago de Chile: RIL Editores / Universidad Autónoma de Chile. https://doi.org/10.32457/ISBN9789568454951982020-ED1

Sandoval-Obando, E. y Sandoval-Díaz, J. (2020). Psicología de la emergencia en contexto de pandemia: aportes y herramientas para la intervención psicológica. Revista Tesis Psicológica, 15(2) 1-32 https://doi.org/10.37511/tesis.v15n2a14

Strauss, A. y Corbin, J. (2002). Bases de la investigación cualitativa. Técnicas y procedimientos para desarrollar la teoría fundamentada. Universidad de Antioquia. https://diversidadlocal.files.wordpress.com/2012/09/basesinvestigacion-cualitativa.pdf

Villar, F. (2012). Successful ageing and development: the contribution of generativity in older age. Ageing and Society, 32(7), 1087--1105. https://doi.org/10.1017/S0144686X11000973

Villareal, M., Hernandiz, J., Leal, C., Medina, S. y Vásquez, C. (2020). Bienestar psicológico y generatividad en adultos mayores que practican el voluntariado. Neurama, revista electrónica de psicogerontología 7(1), 4653. http://46.29.49.21/ creanete/neu/articulos/neuramavol7 1.pdf\#page=45

Wells, M. (2010). Resilience in older adults living in rural, suburban, and urban areas. Online Journal of Rural Nursing and Health Care, 10(2), 45-54. https://rnojournal.binghamton.edu/index.php/RNO/article/view/55/46 